\title{
Aspekte der Geschichte westeuropäischer Lehrerbildung
}

\begin{abstract}
Summary: The history of teacher education is very multi-faceted; therefore it is only possible to point to highlights of its progression. This article indicates some developmental aspects of the history of teacher education in Germany and gives some brief sketches of the history of teacher education in Austria, France, and England, with the intent to provide insight into current educational-policy discussions and developments.
\end{abstract}

Резюме: История подготовки учителей разнообразна настолько, что всякий раз можно лишь фрагментарно рассмотреть ее течение. На основе истории подготовки учителей в Германии, а также в кратких зарисовках из истории подготовки учителей в Австрии, Франции, Англии и Италии в данной статье показываются некоторые линии развития, которые делаю более понятными современные образовательно-политические дискуссии.

Zusammenfassung: Die Geschichte der Lehrerbildung ist so vielfältig, dass immer nur Schlaglichter auf ihren Verlauf geworfen werden können. Anhand der deutschen Geschichte der Lehrerbildung sowie in kurzen Abrissen auch der Geschichte der Lehrerbildung in Österreich, Frankreich, England und Italien werden in diesem Artikel einige der Entwicklungslinien gezeigt, die die heutigen bildungspolitischen Diskussionen und Entwicklungen verständlicher machen sollen.

\section{Aspekte der Geschichte der Lehrerbildung in Deutschland}

\author{
Kurzer Abriss der Schulentwicklung in Deutschland \\ vom Mittelalter bis zur Aufklärung
}

Die Schulentwicklung war zunächst eingebunden in den europäischen Christianisierungsprozess. Im Mittelalter übernimmt die Schule in Form von Domschulen (auch Stifts-, Kloster- oder Klerikerschulen genannt) die Aufgabe der Ausbildung von kirchlich geprägten Amtsfunktionen. Die Domschulen bildeten Kleriker aus, die zugleich Lehraufgaben übernahmen sowie Verwaltungsbeamte, Juristen und später auch Mediziner. Gelehrt wurde in der Regel in der lateinischen Sprache, nach dem Lehrplan der "Septem Artes Liberalis“ (Grammatik, Rhetorik, Dialektik, Arithmetik, Geometrie, Musiktheorie und Astronomie). Dabei wurde von einem statischen Welt- und Wissenschaftsverständnis ausgegangen d.h., was der Mensch überhaupt wissen konnte, war bekannt, worauf es erzieherisch und unterrichtlich ankam, war Einübung und Nachsprechen: „Die Schule ist vor allem Buchschule, ihr Lernen ist bestimmbares, planbares und kontrollierfähiges Buchlernen“, schreibt Keck $(1997,188)$.

Im Übergang vom Mittelalter zur Neuzeit (Ende 15. Jahrhundert, Anfang 16. Jahrhundert) griff die sogenannte Renaissance auf die Werte der Antike zurück und das Bedürfnis nach einer rein weltlichen Bildung wurde gefördert. Auch durch das allmähliche Aufblühen des Handels und der Hanse wurde es im ausgehenden Mittelalter immer notwendiger, die Muttersprache, die Sprachen der Nachbarländer und das Rechnen zu erlernen. Dem Zeitalter der Renaissance liegt der Humanismus zugrunde, der eine umfassende Bildungsreform wollte, die es ermöglichte, dass sich die menschlichen Fähigkeiten durch die Verbindung von Wissen und Tugend entfalten können. Durch Nachahmung klassischer Vorbilder wollte man ein ideales Menschentum verwirklichen. Die Domschule entwickelte sich daher weiter von einer klerikalen und aristokratischen zu einer 
bürgerlichen und demokratischen Schule, einer humanistischen Gelehrtenschule, die nicht mehr nur von Jugendlichen des Adels sondern auch vom Bürgertum besucht wurde (Blankertz 1982, 19).

Gegen Ende des Mittelalters wurde erstmals die dominierende Rolle der katholischen Kirche in Staat und Gesellschaft infrage gestellt. Die Reformation leitete den größten Wandel in diese Richtung ein (Keck, 2009, 159). Unter Martin Luther (1483 bis 1546) als Begründer der Reformation begann im 16. Jahrhundert eine Spaltung des Christentums in verschiedene Konfessionen. Viele Gebiete des Heiligen Römischen Reiches deutscher Nation wurden protestantisch und katholischer Besitz ging vielerorts in den Besitz der Fürsten über. Luther ging gegen den Autoritätsanspruch der Kirche des Papstes vor und vertrat die These, dass der Glaube allein und nicht Sakramente und Buße Gnade vor Gott erwirken. In der Kirche Luthers stand im Gottesdienst nicht wie in der katholischen Messe, die heilige Handlung der Verwandlung von Brot und Wein in den Leib und das Blut Christi im Mittelpunkt, sondern die Predigt und der Katechismusunterricht.

Jeder sollte daher in der Schriftsprache unterwiesen werden, um das „Gottes Wort“ in der Bibel in der Muttersprache lesen zu können. Lutter hatte die vorher nur lateinischer Sprache vorhandene Bibel ins Deutsche übersetzt. Die Erfindung der Buchdruckerkunst (Mitte des 15. Jahrhunderts) ermöglichte eine weite Verbreitung der Bibel. Zunächst mussten die Erwachsenen das Lesen erlernen, um diese Fähigkeit dann an die Kinder weiterzugeben. Es entstand die "gemeine Schule“, auch Küster- oder Meßnerschule genannt (Keck, 2003, 22). Damit war der Weg geebnet für die Alphabetisierung des Volkes und die Institutionalisierung von Lese- und Schreibtechniken, die durch Vorsagen und Nachsagen nicht mehr nur sonntags in der Kirche, sondern täglich im Unterricht gelehrt werden sollten.

Der Katechismus (Handbuch der Unterweisung in Grundfragen des christlichen Glaubens) war die fundamentale Glaubensgrundlage für beide Konfessionen und bedingte letztlich eine allgemeine, von der Konfession unabhängige Alphabetisierung. Daher wird Bildung nicht nur von Luther sondern auch von den Jesuiten als Vertreter des Katholizismus als das Fundament aller Reform betrachtet (Keck, 2003, 25). Sowohl Reformation als auch Gegenreformation setzen damit erste Anfänge für ein Volksschulwesen, denn ab dieser Zeit wurde ein systematischer Religionsunterricht notwendig, für den das Lesen und Schreiben erforderlich war (Blankertz, 1982, 20).

Im 17. Jahrhundert, der Zeit der Nachreformation und dem Zeitalter des Absolutismus, wurden die Landesfürsten und freien Reichsstädte zu Schulträgern mit je eigenen Schulordnungen wie z.B. Gotha 1642 mit einer für jeden einzelnen Staat zentralen Schulplanung (Keck 2002, 1112). Erst ab dieser Zeit der territorialen Konfessionsausbildung, (d.h. der jeweilige Fürst bestimmt die religiöse Ausrichtung seiner Untertanen) beginnen sich protestantische und katholische Schulsysteme zu unterscheiden (Keck, 2003, 26). Die Schulordnungen führten dazu, dass nun über die Struktur von Unterricht und Schulorganisation über die Landesgrenzen hinaus diskutiert wurde. Der Schulmethodus von Gotha galt schon zu seiner Zeit als Praxismodell. Im Mittelpunkt jeder Unterweisung stand jedoch neben der Gottesfurcht der Gehorsam gegenüber dem Landesfürsten.

Bezüglich der Lehrerbildung für das höhere Lehramt muss man rekapitulieren, dass bis zu Beginn des 19. Jahrhunderts in den Latein- und Gelehrtenschulen katholische oder protestantische Geistliche unterrichteten. Die Lehrer für das höhere Lehramt hatten immer eine universitäre wissenschaftliche Ausbildung, auch wenn diese nicht auf das Lehren, sprich Didaktik und Pädagogik, ausgerichtet war. Die Reform der Universität, die auf Wilhelm von Humboldt (17671835) zurückgeht, veränderte auch das Bild des Gymnasiallehrers, der jetzt die Gymnasiasten auf das zukünftige Universitätsstudium vorbereiten sollte. Die Ausbildung an der Universität wurde 
deshalb auf die Fachwissenschaften orientiert. Dieses zeigte sich auch in der Einführung der berufsqualifizierenden Staatsprüfung 1810 (examen pro facultate docendi) und der Abiturprüfung 1812 in Preußen.

Damit trennt sich erstmals die Professionalisierung des höheren Lehramts von der Theologie. Die Prüfungen wurden zunächst von der Philologie bestimmt, doch allmählich werden auch Mathematik und die Naturwissenschaften bedeutsam. Durch die preußische Prüfungsordnung von 1831 und die Neuregelung von 1866 löst der fachwissenschaftlich gebildete Gelehrte den umfassend gebildeten Schulmann ab. Pädagogik ist nach wie vor kein Pflichtfach - die Überzeugung, ein guter Wissenschaftler sei immer auch ein guter Lehrer, wird bis weit in die Jetztzeit hinein gepflegt (Sandfuchs, 2004, 17ff.).

Eine umfassende Volksbildung und eine institutionalisierte Lehrerausbildung für das niedere Schulwesen gab es jedoch lange Zeit noch nicht. Man muss betonen, dass - trotz bedeutsamer Modelle und Schriften von Johan Amos Comenius/Komensky (1592-1670), Wolfgang Ratke (15711635) und Andreas Rhyer (1601-1669), die von Intellektuellen rezipiert wurden - die Schulsituation auf dem Land in einem erbärmlichen Zustand war, sofern es überhaupt Schulen gab. Eine Gruppe von Kindern allen Alters wurde in der Wohnstube des „Schulmeisters“ unterrichtet, in der sich auch die Familie und das Vieh aufhielt. Obgleich es eine Unterrichtspflicht gab, gingen die meisten Kindern nur im Winter und sonntags zur Schule, da sie in ihren Familien zur täglichen Feldarbeit gebraucht wurden. Die Lehrer für das Volk hatten keine Ausbildung, sie rekrutierten sich häufig aus entlassenen Soldaten, einfachen Handwerkern und Arbeitslosen, die selbst kaum rechnen und lesen konnten.

Von 1729 gibt es z.B. Berichte über den Bewerbungsvorgang für eine Schulstelle vor der Gemeinde, bei der der Bewerber seine Kenntnisse bezüglich der Kirchenlieder, des Lesens des Katechismus, des Schreibens und des Rechnens zeigen musste.

\footnotetext{
„Jakob Moehl, Weber, hat die 50 Jahre hinter sich. Er sang: O Mensch beweine, Zeuch ein zu deinen Thoren. Wer nur den lieben Gott läßt walten. Melodie ging ab in viele andere Lieder, er quiekte manchmal, so doch nit sein soll. Gelesen Josua 19, 1-17 mit 10 Fehlern, buchstabierte Josua 18, 26-29 ohne Fehler. Dreierlei Handschriften gelesen - schwach und mit Stocken. Drei Fragen aus dem Verstand - befriedigend. Aus dem Katechismo die 10 Gebote und die 41. Frage aufgesagt, ohne Fehler, drei diktierte Reihen geschrieben, 5 Fehler, des Rechnens ist er nit kundig." Jakob Moehl wurde von der Gemeinde und dem Pastor einstimmig gewählt, weil die anderen Kandidaten noch schlechter waren" (zitiert nach „Die kaufmännische Schule“, H. 12/1976).
}

Dieser Zustand änderte sich allmählich mit der Epoche der Aufklärung, einer Epoche der modernen westlichen Philosophie. Man kann auch vom ,Zeitalter der Aufklärung' sprechen. Der Philosoph Emanuel Kant beantwortet, als die Epoche bereits ihrem Ende entgegenging, die Frage „Was ist Aufklärung?" wie folgt:

\begin{abstract}
„Aufklärung ist der Ausgang des Menschen aus seiner selbst verschuldeten Unmündigkeit. Unmündigkeit ist das Unvermögen, sich seines Verstandes ohne Leitung eines anderen zu bedienen. Selbstverschuldet ist diese Unmündigkeit, wenn die Ursache derselben nicht am Mangel des Verstandes, sondern der Entschließung und des Muthes liegt, sich seiner ohne Leitung eines andern zu bedienen. sapere aude! Habe Muth dich deines eigenen Verstandes zu bedienen! Ist also der Wahlspruch der Aufklärung“ (Kant, 1784, 169). „... es ist so bequem, unmündig zu sein...“ (Kant, ebd.).
\end{abstract}

Die Idee des vernunftgeleiteten, mündigen und zum Handeln berufenen Menschen wirkte sich auch auf die Pädagogik aus, denn der Kindererziehung und damit auch dem Schulwesen wurde eine zentrale Rolle bei der Entwicklung zum mündigen Bürger zugewiesen. Schule erhielt zunehmend das Ausbildungsmonopol in Bezug auf Vermittlung von Wissen, Können und Werten. „Sie, die Vernunft, galt als ein und dieselbe für alle denkenden Subjekte, für alle Nationen, Rassen, Epochen 
und Kulturen (...). Das finden wir, wie selbstverständlich, auch bei einem Klassiker der Pädagogik: Jean-Jacques Rousseau“, schreibt Blankertz $(1982,27)$. Neu war nun, dass Erziehung in der Hand des Menschen liegt und zum Gegenstand einer eigenen Reflexion gemacht werden muss. Das zeigt sich darin, dass Christian Trapp, ein Theoretiker der Aufklärungspädagogik und Philanthrop, 1779 den ersten Lehrstuhl für Pädagogik an einer deutschen Universität in Halle erhielt und Johann Heinrich Campe (1746-1818) 16 Bände herausgab mit dem Titel: „Allgemeine Revision des gesamten Schul- und Erziehungswesens von einer Gesellschaft praktischer Erzieher". Er berief sich dabei vor allem auf Rousseau, indem die befreiende Wirkung seiner Pädagogik und die Anerkennung des Eigenrechtes des Kindes hier schulpädagogisch konkretisiert wurden. Die Philanthropine (Philanthrop griech. ,Menschenfreund') waren eine Alternative zu der im Schematismus erstarrten lateinischen Schulgelehrsamkeit. Sie hoben viele einengende Gebräuche auf wie z.B. das Tragen von Perücken. Statt nur über Büchern zu sitzen, gab es Sport und Wanderungen. Den Kern der Didaktik machte eine weltoffene Bildung aus, die - an der Schwelle zum Zeitalter der Industrialisierung - an modernen Sprachen, Mathematik und den Naturwissenschaften orientiert war. Die Einrichtungen der Philanthropen zeigten, dass „Schule auch Stätte der Freude, des Frohsinns und Spiels sein könne, dass Schule nicht gekennzeichnet sein müsse durch Schreie gezüchtigter Kinder und vom Zorn berauschter Lehrer“ (Blankertz, 1982, 81). Obgleich mehr als 60 reformoffene aufgeklärte Erziehungs-, Unterrichts- und Lehranstalten zwischen 1770 und 1800, ausgehend von dem von Johann Bernhard Basedow (1724-1790) gegründeten Philanthropin in Dessau, in Deutschland eingerichtet wurden, hat sich diese Pädagogik nicht durchgesetzt (vgl. etwa Feige, 1997, 4). Die meisten dieser Anstalten überlebten kaum länger als zwanzig Jahre. Bewirkt haben die Philanthropen jedoch, dass nun eine allgemeine Unterrichtspflicht von staatlicher Seite lückenlos realisiert werden sollte und der Staat ausreichend Schulen zur Verfügung stellen musste, in der die Kinder und Jugendlichen auf einen Beruf im Sinne von Nachwuchsbildung vorbereitet werden sollten (Blankertz, 1982, 86f.). Das „Generallandschulreglement" von 1763 in Preußen enthielt die Aufforderung zum Schulbesuch und bildete damit die Grundlage für die Entwicklung des deutschen Volksschulwesens.

\section{Entwicklungsstufen der Lehrerbildung}

Nach dem Generallandschulreglement beginnt nun auch die Zeit, in der man von einer allmählichen Professionalisierung des Lehrers für das niedere Schulwesen, die Volksschule, sprechen kann. Die Entwicklung der deutschen Volksschullehrerausbildung kann in drei Phasen gegliedert werden (vgl. Oelker \& Neumann, 1985, 127):

- Schulung im Lehrerseminar

- Studium in pädagogischen Akademien (später hießen sie Pädagogische Hochschulen):

- Wissenschaftliche Ausbildung an Universitäten

\section{Lehrerseminar}

Erste Vorläufer eines Lehrerseminars gab es bereits im 17. und 18. Jahrhundert. Zu den Aufklärungspädagogen gehörten neben den Philanthropen auch die Pietisten. Nach Keck kommt insbesondere durch den Pietismus die Bildung des Volkes in den Blick (Keck, 1989, 199). August Hermann Francke (1663-1727), der bedeutendste Pädagoge unter den Pietisten, gründete 1694 in Halle an der Saale eine Armenschule, die sich bald zu einer Anstalt entwickelte, die neben der Schule Spinnereien, Webereien, eine Apotheke und eine Druckerei enthielt. Der Pietismus lehrte im Gegensatz zu Luthers Protestantismus, dass der Mensch, der sein Leben Gott weihe und gute Werke 
vollbringe, von Gott nicht im Stich gelassen werde. Francke war geprägt durch die Schulordnung von Gotha von 1642 und zielte auf eine beruflich orientierte realistische Wendung des Schulwesens ab. Der Pietismus forderte die Trennung von Staat und Kirche und half, einen neuen Stand des gebildeten Bürgertums zu schaffen, der das Zentrum der deutschen Kultur wurde (Blankertz, 1982, 54). „Erziehung führt in das wirkliche Leben, und das wirkliche Leben erfordert ausdrücklich Erziehung“ (Blankertz, 1982, 28). Aus diesem Gedankengut heraus gründete Johann J. Hecker (1707-1768), ein Schüler Franckes, 1748 das erste preußische Lehrerseminar, ein „Küster- und Schulmeisterseminar", das spezifisch Lehrer für die von ihm ins Leben gerufene "Realschule“ ausbilden sollte. Diese Lehrerseminare, die sich zunehmend weiter verbreiteten, waren zunächst keine eigenständigen Einrichtungen, sondern sie waren Waisenhäusern, Bürgerschulen oder Lateinschulen zugeordnet (vgl. Sandfuchs, 2004, 22). Erst ab Anfang des 19. Jahrhunderts wurden die Lehrerseminare eigenständige Institutionen.

Im Laufe des 19. Jahrhunderts war es dann auch möglich, dass Frauen in den Volksschulen unter-richteten. Das erste preußische Lehrerinnenseminar wurde 1832 gegründet. Die Gründe dafür waren Lehrermangel bzw. Lehrerüberfluss. Lehrerinnen durften nicht heiraten und erhielten nur Zeitverträge, so dass sie schnell eingestellt bzw. entlassen werden konnten. Die Ausbildung von Frauen und Männern war bis in die 1920er Jahre getrennt.

Adolf Diesterweg (1790-1866) war von 1832 bis 1847 Direktor des Stadtschulseminars in Berlin. Als liberaler Pädagoge engagierte er sich für eine professionalisierte Volksschullehrerbildung und die soziale Anerkennung des Volksschullehrers, der zu dieser Zeit eher verachtet wurde als Hungerleider, da er einen nur geringen Lohn erhielt sowie als Vertreter der „Prügelpädagogik“ angesehen wurde. Diesterweg war Anhänger Johann Heinrich Pestalozzis (1746-1827) und er trugen viel dazu bei, dessen Ideen und didaktischen Grundsätze zu vertreten, die er mit den Auffassungen von Friedrich Fröbel (1782-1852) verband.

In seiner Zeit als Direktor des Lehrerseminars in Moers (von 1820-1832) besuchte Diesterweg verschiedene Volksschulen, die er zum großen Teil in einem sehr schlechten Zustand antraf.

\footnotetext{
„Schärfste Kritik übte Diesterweg an der Tatsache, daß Ungehorsam und Zuchtlosigkeit den Unterricht an einigen Schulen bestimmen. Die Lehrer seien nicht in der Lage, sich durchzusetzen. Über die von der frühen Industrialisierung erfaßten Städte des Wuppertals fällte er ein besonders hartes Urteil: ,In den meisten Schulen Elberfelds und Barmens herrscht unter den Schülern ein solcher Grad von Unruhe, Unbeständigkeit, zerstreutes Wesen, Frechheit, Ruhelosigkeit und wahre Gottlosigkeit, daß die Möglichkeit fast übertroffen wird'. So habe in der Schule des Lehrers Wilms, einem pensionsreifen alten Mann, ,ein wilder Tumult mit Apparaten, Füßen und Armen, ein tolles Durcheinanderschreien einer ungezogenen, frechen Jugend' geherrscht. Das Ganze habe geradezu den Anblick eines offenen Kriegszustandes dargeboten. Die Lehrer müssten sich mit Drohung und Gewaltanwendung Respekt verschaffen." (Goebel, 1995, 103).
}

Diesterweg machte für diese Zustände die unzureichende Seminarausbildung verantwortlich, auch die seiner eigenen Seminaristen. „Der Hebel der Schulreform, die den methodisch besser ausgerüsteten Lehrer zum Ziel hatte, war also bei den eigenen Seminarabsolventen anzusetzen“, schreibt Goebel $(1995,104)$. In einem der Seminare fand Diesterweg "das Bessere, welches das Seminar zu verbreiten sucht, eingeführt: sinnvolles Lesen, verständlichen und gemüthlichen Religionsunterricht, wohlklingendes sanftes Singen, überhaupt denkendes Lehren und Lernen, geregelte Zucht und unbedingten Gehorsam" (ebd., 104). Allerdings muss man auch sehen, dass die Schulverhältnisse häufig unzumutbar waren. Die Räume waren für die große Zahl an Schülern teilweise 160 Kinder - viel zu klein und mangelhaft ausgestattet. Die Kinder mussten auf den Knien schreiben, da keine Tische aufgestellt werden konnten. Die Lehrer mussten 40 Wochenstunden arbeiten, die Lehrerbesoldung war so gering, dass sie mehrere Nebenberufe (Küster, 
Versicherungsvertreter, Kleinbauer) annehmen mussten, um leben zu können. Oft erhielten sie von den Eltern Fleisch, Eier und Mehl. [Erstaunlicherweise habe ich als junge Lehrerin um 1970 in der Volksschule auf dem Land ebenfalls noch Fleisch und Eier von den Bauern als Geschenk erhalten. So lange hielt sich diese Tradition.]

Der politische Hauptgrund für die sehr viel langsamere Entwicklung der Volksschullehrerausbildung im Vergleich zur Professionalisierung im Bereich der höheren Bildung, ist nach Oelker und Neumann im Scheitern der Revolution von 1848 zu sehen $(1985,128)$. Reformorientiere und liberale Pädagogen wie u.a. Adolf Diesterweg wurden zum Sündenbock für die Revolution erklärt, eingesperrt oder - wie Diesterweg - ihres Amtes enthoben. „Seit Beginn des 19. Jhds. wird Schulreform zunehmend vom Staat zur Steuerung der gesellschaftlichen Entwicklung in Anspruch genommen", schreibt Keck $(2009,161)$. Dies zeigt sich insbesondere in der Reaktion auf die Revolution von Seiten des Wilhelminismus (Kaiser Wilhelm): Zementierung des Ständestaates und Aufbau von Bildungsprivilegien für Adlige und höhere Stände.

Allerdings hatten die Zerschlagung der Revolution und die darauf folgenden Reglementierungen auch etwas Gutes insofern, als erste Standards für die Volksschullehrerbildung entwickelt wurden. Ab 1890 wurden dann flächendeckend Lehrerseminare eingerichtet (in kleinen Städten, um die Kandidaten von aufrührerischen Bewegungen abzuschotten) und diese eine Ausbildung erhielten, auch wenn der Wissenskanon, der hier erworben werden sollte, nach wie vor sehr eng war. Die „Allgemeinen Bestimmungen“ des Volksschul-, Präparanden- und Seminarwesens von 1872, die für Preußen Gültigkeit hatten, enthalten nun eine Lehrordnung und einen Lehrplan (Oelkers \& Neumann, 1985, 128). Von der Umsetzung der Forderung, die u. a. Diesterweg schon 1836 erhob, dass auch die Lehrerausbildung für die Volksschule einer universitären Bildung bedürfe, da die Seminarausbildung nicht dem gegenwärtigen Stand der Wissenschaft entspräche, war man nach wie vor sehr weit entfernt.

\section{Studium in pädagogischen Akademien}

Ob die Universität im Stande ist, Lehrer auszubilden, die ihr Lehrgeschäft verstehen sollen, also eher praktisch ausgebildet werden müssen, wurde schon Mitte des 19. Jahrhunderts von Lehrerverbänden auf ihren Versammlungen diskutiert (vgl. Sandfuchs 2004, 25) und diese Diskussionen sind nie ganz verstummt. Auf der Lehrerversammlung in Königsberg 1904 wurden z. B. folgende Beschlüsse gefasst (vgl. Sandfuchs, 2004, 29):

1. Die Universität als Zentralstelle wissenschaftlicher Arbeit ist die geeignetste, durch keine andere Einrichtung vollwertig zu ersetzende Stätte für die Volksschullehrerbildung.

2. Für die Zukunft erstreben wir daher die Hochschulbildung für alle Lehrer.

3. Für die Jetztzeit dagegen fordern wir, daß jedem Volksschullehrer aufgrund seines Abgangszeugnisses vom Seminar die Berechtigung zum Universitätsstudium erteilt werde.

1909, noch vor dem ersten Weltkrieg, machte der Deutsche Lehrerverein den Kompromissvorschlag einer „Pädagogischen Akademie“.

Welche kontroversen Diskussionen dann auf der Reichsschulkonferenz von 1919 geführt wurden, zeigt z.B. die Einlassung von Eduard Spranger, einem Klassiker der modernen Pädagogik. Er lehnte das Abitur für Volksschullehrer ab und warnte vor einem „Ansturm der Volksschullehrer auf die Universitäten“. Andere Pädagogen hielten dagegen, dass die Pädagogik mehr als bisher der „Fundamentierung auf exakter wissenschaftlicher Forschung“ bedürfe (Sandfuchs, 2009, 64f.). Pädagogik wurde als eine hermeneutische Wissen-schaft verstanden, die die 
Erziehungswirklichkeit im Verstehensprozess durchdringen sollte. „Praxis-druck einerseits und Akademisierung andererseits bestimmten das theoretische Bemühen der Pädagogik während der Weimarer Republik" (Weiner, 1992, 203). Hermann Nohl, einer der Hauptvertreter der geisteswissenschaftlichen Pädagogik, wollte insbesondere die Lehr- und Forschungstätigkeit eng auf die Anforderungen der Praxis in allen Bereichen beziehen (ebd., 204). Doch gerade auch die Frage nach der Gewichtung von Theorie und Praxis wurde kontrovers diskutiert.

1926 gründete der damalige preußische Kultusminister gemäß der Weimarer Verfassung die erste „Pädagogische Akademie“ bzw. „Pädagogische Hochschule“, für deren Aufnahme das Abitur Voraussetzung war. In Baden-Württemberg und Bayern (hier schon 1910) wurden - meist konfessionell gebundene - „Lehrerbildungsanstalten“ eingerichtet, für deren Aufnahme das Abitur nicht immer zwingend war. Diese „Zwischenstufe“ zwischen seminaristischer und universitärer Ausbildung wurde damit begründet, dass die Volksschule keine wissenschaftlichen Fachlehrer brauche, sondern in erster Linie Erzieher. Zwischen Grund- und Hauptschule (1. Bis 4. Schuljahr bzw. die oberen Jahrgänge der Volksschule) wurde dabei nicht unterschieden. Die pädagogischen Akademien stellten folgerichtig nicht die wissenschaftliche Forschung, sondern die umfassende, vor allem auch ästhetisch-musisch ausgerichtete Bildungserfahrung in den Mittelpunkt der Berufsvorbereitung (vgl. Oelker \& Neumann, 1985, 129). Die Standesvertreter der Volksschullehrerschaft jedoch waren anderer Meinung und forderten die gleiche wissenschaftliche Vorbildung für aller Lehrämter, für Volksschule ebenso wie für Gymnasien. Doch bis heute wird diese Forderung in Deutschland nicht erfüllt.

Aus der Volksschullehramtsausbildung heraus entstanden zu Beginn des 20. Jahrhunderts weitere Lehrämter wie das Lehramt des Realschullehrers, des Hilfsschullehrers und des Berufsschullehrers. Auch hier wird die Akademisierung der Ausbildung angestrebt (vgl. Sandfuchs, 2004, 25). Auf diese Lehrämter kann jedoch an dieser Stelle nicht weiter eingegangen werden.

\section{Zeit des Nationalsozialismus}

Die nationalsozialistische Schulpolitik brachte in Bezug auf die Organisation keine schwerwiegenden Einschnitte. Die Erziehung der NSDAP konzentrierte sich auf Jugendorganisationen wie die Hitlerjugend, den Aufbau der "Staatsjugend" und entsprechende Schulungen. Bei der Volksschullehrerbildung dagegen „kulminierten Antiintellektualismus und restriktive Schulpolitik" (Blankertz, 1982, 242). Alle jüdischen Lehrerbildungsstätten wurden geschlossen und im "Gesetz gegen Überfüllung der deutschen Schulen und Hochschulen“ wird der Eindruck suggeriert, als gäbe es einen überproportional hohen Anteil jüdischer Studierender an den Universitäten. 1933 wurden die Pädagogischen Akademien in „Hochschulen für Lehrerbildung“ (HfL) umbenannt, was jedoch nur scheinbar eine akademische Lehrerbildung zu sichern schien. Republiktreue Dozenten wurden entlassen und 1940 wurden die Hochschulen durch „Lehrerbildungsanstalten“ (LBA) ersetzt. Den Einrichtungen waren streng ideologisch durchstrukturierte Internatsbetriebe (für Männer und Frauen getrennt) angegliedert. Schon bald zeichnete sich ein eklatanter Volksschullehrermangel ab - insbesondere ab 1939 auch kriegsbedingt -, so dass begabten Schülern schon nach einem achtjährigen Besuch der Volksschule die Möglichkeit zu einer Ausbildung zum Volksschullehrer angeboten wurde. Abitur war keine Voraussetzung mehr. Die Ausbildungszeit betrug 5 Jahre, mit Mittlerer Reife sogar nur 3 Jahre. Nach zwei Jahren Dienst musste eine zweite Lehrerprüfung abgelegt werden, worauf die lebenslange Verbeamtung erfolgen konnte. Die Ausbildung war kostenlos und damit unabhängig vom finanziellen Status der Eltern. 
Eine große Zahl der Professoren bekannte sich zu Hitler und war bereit, die nationalsozialistischen Erziehungsziele zu übernehmen und eine große Zahl der Lehrerschaft und der Lehrerbildner trat in die NSDAP ein. Teils taten sie es, weil es von ihnen als Beamte erwartet wurde, teils waren Sie aber auch überzeugte Anhänger Hitlers und der Erziehungsvorstellungen des Nationalsozialismus. Dresselhaus schreibt dazu: „Die Erreichung der faschistischen Systeme in Italien und Deutschland beruht auf einer engen Verflechtung der faschistischen Bewegung mit weiten Teilen der herrschenden Klasse. Hierzu gehören vor allem das Militär, die Bürokratie und die Kirchen (...) Aber nicht nur das Militär auch die mittleren und höheren Beamten in Justiz und Verwaltung sowie Lehrer an Hochschulen und Gymnasien hatten in Deutschland dem kaiserlichen Obrigkeitsstaat treu gedient, einem Staat, der den Glanz einer Großmacht ausstrahlte und zugleich ihre gesellschaftlichen Privilegien schützte. Der Faschismus schien ihnen die Ordnung im Inneren und die Machtstellung nach außen wiederherzustellen“ $(1997,410)$. Die Struktur und Ideologie von Bürokratie, Kirche und Lehramt, war durch die vorausgehende Feudalherrschaft autoritär bestimmt und auf Gehorsam ausgerichtet, so dass vielfach Beamte nur allzu bereit waren, das System des Nationalsozialismus zu unterstützen.

In zahlreichen Lehrerbildungsanstalten wurde, wie z.B. in Kiel, die Ernennung Hitlers zum Reichskanzler zusammen mit nationalsozialistischen Studenten im Januar 1933 durch Hissen der Hakenkreuzflagge über dem Gebäude begrüßt. Der Direktor zeigte sich einverstanden mit den personellen Veränderungen, die sich durch das Gesetz zur „Wiederherstellung des Berufsbeamtentums“ (BBG) ergaben, das hieß u.a., dass nur diejenigen, die eine „rein arische Abstammung" nachweisen konnten, im öffentlichen Dienst angestellt werden konnten. „Jüdisch versippte“ Beamte waren in den Ruhestand zu versetzen. ${ }^{1}$ Das veranlasste viele Professoren und Lehrer dazu, aus Angst, den Arbeitsplatz zu verlieren, Mitglied in der NSDAP zu werden, auch wenn sie sich nicht zum Faschismus bekennen konnten. Darüber hinaus gab es wie z.B. in Kiel auch Kollegen, die nicht nur Mitglieder der NSDAP waren, sondern auch Mitglieder der SS und/oder SA. ${ }^{2}$

Da die nationalsozialistische Herrschaft verhältnismäßig kurze Zeit dauerte, sind die Auswirkungen bis heute nur schwer abzuschätzen. „Dennoch wird man davon ausgehen müssen, daß eine ganze Generation von Kindern und Jugendlichen in diesen zwölf Jahren erheblichen Einflüssen totalitärer Herrschaft ausgesetzt und unterlegen war“ (Weiner, 1992, 199).

\section{Lehrerbildung in der Bundesrepublik Deutschland}

Nach 1945 wollten die Besatzungsmächte zunächst ein horizontal gegliedertes Schulsystem in Deutschland sowie eine Lehrerbildung an wissenschaftlichen Hochschulen einführen. Doch es setzte sich das traditionelle dreigliedrige Schulsystem durch. Für die Volksschullehrerbildung etablierten sich in fast allen Bundesländern „Pädagogische Hochschulen“, die in den 1970er Jahren in die Universitäten integriert wurden.

Eine wesentliche Spezifik deutscher Lehrerbildung liegt in der Kulturhoheit der 16 Bundesländer. Ein Mindestmaß an Einheitlichkeit wird durch die Kultusministerkonferenz (KMK) gewährleistet. Dennoch ist nicht immer gesichert, dass Abschlüsse jeweils gegenseitig anerkannt werden.

Seit dem 1970 vorgelegten „Strukturplan zur Neuordnung des Bildungswesens" gliedert sich das Schulsystem auf in die Elementarstufe (Kindergarten und Vorschulerziehung), die vier (in einigen Bundesländer sechsjährige) Primarstufe, die weiterführenden Schulen (Hauptschule, Realschule mit dem Sekundarstufen I - Abschluss der „Mittleren Reife“ und Gymnasium mit dem Abitur

Zitiert nach URL http://www.uni-kiel.de/ns-zeit/bios/glossar.shtml\#beamtengesetz.

Siehe auch URL http://www.uni-kiel.de/ns-zeit/ph/. 
als Abschluss sowie alle Einrichtungen der Berufsausbildung (Sekundarstufe II). Deutschland hat bis heute das dreigliedrige Schulsystem erhalten und in fast allen Bundesländern endet die Primarstufe bereits nach dem 4. Schuljahr - damit unterscheidet es sich von fast allen anderen europäischen Ländern, die entweder eine 6jährige Primarstufe oder ein Einheitsschulsystem haben.

Lehrer für die Primar- und Sekundarstufe I müssen in der Regel ein 6-8semestriges Studium (in den meisten Bundesländern inzwischen konsekutiv (Bologna-Prozess) und für das Lehramt für die Sekundarstufe II ein 10semestriges Studium absolvieren. Das heißt, dass es nach wie vor unterschiedliche Lehramtsausbildungen gibt, was sich auch im Lehrergehalt niederschlägt. Grundund Hauptschullehrer erhalten ein geringeres Gehalt als Gymnasiallehrer. In der Regel werden zwei Unterrichtsfächer und Bildungswissenschaften (Pädagogik und Psychologie) studiert. In der Ausbildung für das gymnasiale Lehramt gewinnt die Pädagogik zunehmend an Bedeutung. Während des Studiums werden mehrere schulpraktische Studien absolviert. Nach dem ersten Staatsexamen bzw. dem Masterdiplom (das von der Universität vergeben wird) folgt eine ein- bis zweijährige Referendariatszeit bzw. ein Vorbereitungsdienst, der der praxisorientierten Berufsvorbereitung dient und in der meist auch schon eigenverantwortlich unterrichtet wird. Die Ausbildung wird mit einem zweiten Staatsexamen abgeschlossen. Die Lehrkräfte sind zum lebenslangen Lernen durch Weiterbildung verpflichtet.

Die Chance, eine Planstelle an einer Schule zu erhalten, hängt vom zyklisch wiederkehrenden Überfluss oder Mangel an Lehrkräften ab. Da die Lehrerbildung ständigen Reformen unterworfen ist, sind diese Ausführungen nur Richtmaße.

„Der lange Marsch der Lehrerausbildung vom voraussetzungslosen Nebenberuf zur akademischen Profession ist zunächst als Erfolg zu betrachten (...), jedenfalls wenn man die mehr als hundertjährigen Postulate der Lehrerorganisationen zugrunde legt: Die Bezahlung der Lehrer ist angemessen, die Ausrüstung der Schulen und Universitäten ist besser als je zuvor, traditionelle Minderwertigkeitsgefühle sind verschwunden, lähmende Streitpunkte (zum Beispiel Konfessionalität oder Koedukation) sind kein Thema mehr. (...) Ein differenziertes Angebot an Wissensbeständen hat die deutsche Lehrerschaft in ihrer langen Geschichte nie gehabt.“ (Oelker \& Neumann, 1985, 130).

Die Autoren weisen jedoch schon in den 1980er Jahren darauf hin, dass es offensichtlich keinen Konsens darüber gibt, welches Wissen und in welcher Form Wissen Lehrern vermittelt werden soll, damit sie es sinnvoll anwenden können. Das Theorie-Praxis Problem ist schwer aufzulösen, da die Erwartungen an eine praktische Berufsausbildung und an ein wissenschaftliches Studium unvereinbar zu sein scheinen (vgl. ebd., 131). Zwei gegenläufige Konzeptvorschläge werden derzeit diskutiert: die Vereinheitlichung der Lehrerausbildung auf allen Stufen (Primar- und Sekundarstufen) und das Stufenlehrermodell (inhaltliche Differenzierung nach Primarstufe, Sekundarstufe I und Sekundarstufe II). Die letzten drei Jahrzehnte sind demnach gekennzeichnet durch kontrovers geführte Diskussionen, durch Umstrukturierungen und sich stetig ändernde Reformen. Seit den 1990er Jahren gibt es in allen Bundesländern wieder eine schulformbezogene Ausbildung, wobei in einigen Bundesländern wie u.a. Niedersachsen Primar-, Haupt- und Realschule zusammengefasst werden, während die gymnasiale Lehrerbildung, die Berufsschullehrerbildung und die Sonderschullehrerbildung jedoch getrennte Studiengänge sind.

Was ist nun spezifisch deutsch? Karl Marx schreibt: „Die Deutschen haben in der Politik gedacht, was die anderen Völker getan haben" $(1976,385)$. Damit bezeichnet er eine Besonderheit der Deutschen, ins „Theoretisieren“ zu flüchten (Dresselhaus, 1997, 428). Dies hat nach Dresselhaus geistesgeschichtliche Wurzeln in der Verinnerlichung des obrigkeitsstaatlichen Denkens im Protestantismus von Luther, aus der langfristig auch die Treue des Beamten resultierte. Weiter heißt es bei Marx: 


\begin{abstract}
„Luther hat allerdings die Knechtschaft aus Devotion besiegt, weil er die Knechtschaft aus Überzeugung an ihre Stelle gesetzt hat. Er hat den Glauben an die Autorität gebrochen, weil er die Autorität des Glaubens restauriert hat. Er hat die Pfaffen in Laien verwandelt, weil er die Laien in Pfaffen verwandelt hat. Er hat den Menschen von der äußeren Religiosität befreit, weil er die Religiosität zum inneren Menschen gemacht hat. Er hat den Leib von der Kette emanzipiert, weil er das Herz an die Kette gelegt." (Marx, 1976, 386)
\end{abstract}

Die aus Frankreich kommende revolutionäre Welle wurde 1848 in Deutschland niedergeschlagen, da das deutsche Bürgertum „die von unten nachdrängenden Arbeitermassen mehr“ fürchtete „als die feudale Reaktion“ (Dresselhaus, 1997, 429). Damit versucht Dresselhaus m. E. zurecht die doch eher konservative Schulpolitik, wie sie in Deutschland gegenüber anderen europäischen Ländern noch heute vorzufinden ist, zu erklären.

\title{
Geschichte der Lehrerbildung in anderen westeuropäischen Ländern
}

\section{Österreich}

Im ausgehenden 18. Jahrhundert, im Zuge der Aufklärung, bildeten sich in Österreich und damit u. a. auch in Ungarn, Bulgarien, Rumänien, Tschechien und ebenso in Italien, das sich in dieser Zeit an die Entwicklung in der österreichisch besetzten Lombardei orientierte (Polenghi, 2012, 139), erste Lehrerbildungsanstalten, die sogenannten „Normalschulen“. In Wien wurde die erste dieser Schulen 1771 gegründet. Ziel war eine stärker pädagogisch-wissenschaftlich akzentuierte Berufsvorbildung (Grimm, Bali \& Pirka, 2012, 92). Das Gründungsdokument der österreichischen Volksschule war die von Maria Theresia (1717-1780) unterzeichnete „Allgemeine Schulordnung für die deutsche Normal-, Haupt- und Trivialschule in sämmtlichen Kayserl. Königl. Erbländern“. Es bestimmte Organisation, Inhalt und Methode des Elementarunterrichts für fast ein Jahrhundert (ebd., 92). In ihr wurden "Normen für die Lehrerbildung" formuliert, die für die Normalschulen bindend waren. Anfangs waren es nur dreimonatige "Präparandenkurse“, 1806 wurden sie auf sechs Monate verlängert. Im Vordergrund stand das „Abrichten“ und „Präparieren“ der Zöglinge, doch der Anfang für eine systematische Berufsvorbereitung war gesetzt.

Länger als in Deutschland blieb das höhere Lehramt bis 1848 in den Händen der Theologen wie der Jesuiten und Benediktiner. Pädagogisch-didaktische Aspekte fehlten in dieser Ausbildung. 1876 wurde in Wien das erste "Pädagogische Seminar“ eingerichtet. Die Prüfungsordnung für Kandidaten des Lehramtes an Gymnasien, Realgymnasien und Realschulen von 1911 sah eine „philosophisch-pädagogische Vorprüfung“ am Ende des fünften Semesters vor, dennoch wurde das pädagogische Begleitstudium wie auch in Deutschland nicht in das fachwissenschaftliche Studium integriert.

Die Revolution von 1848 ebnete zwar den Weg für eine grundlegende Modernisierung des gesamten österreichischen Bildungswesens (ebd., 94), doch der Primarbereich blieb zunächst unverändert. Die Präparandenkurse wurden jedoch von einem auf zwei Jahre erweitert. Erst mit dem "Reichsvolksschulgesetz" von 1869 kam es zu einer Verbesserung der „Pflichtschullehrerbildung" und 1875 wurden vierjährige Lehrerbildungsanstalten (LBA) eingerichtet, die staatlich oder katholisch waren. „Damit ging die ziemlich genau 100 Jahre währende Ära der Präparandenkurse zu Ende" schreiben Grimm, Bali und Pirka (ebd., 95). Für die Aufnahme in die LBAs musste das 15. Lebensjahr beendet, sittliche Unbescholtenheit nachgewiesen und die Bürgerschule, das Untergymnasium oder die Unterrealschule abgeschlossen sein. Das Curriculum war von Theorie und Praxis geprägt. Die LBA wurden mit einer "Reifeprüfung" abgeschlossen, die die Zöglinge zum Unterricht an Volksschulen befähigte. 
Mit der Konstituierung der demokratischen Republik Österreich 1918 begann - wie auch in Deutschland zu dieser Zeit - eine neue Ära der Bildungspolitik. Auch hier wurde nun die Akademisierung der „Pflichtschullehrer" gefordert. Es wurde eine hochschulmäßige Ausbildung der Lehrer aller Schultypen an Universitäten mit angeschlossenen Übungsschulen gewünscht (ebd., 98). Doch die Vertreter der bisherigen vierjährigen LBAs setzten sich durch. Der Anschluss Österreichs an das nationalsozialistische Großdeutsche Reich 1938 bis 1945 vereinte die deutsche und österreichische Entwicklung im Bereich der Volksschullehrerausbildung. 1962 erfolgte der Durchbruch zur akademischen Lehrerbildung. Es wurde eine vierjährige Lehrerbildung an Pädagogischen Akademien obligatorisch, die weder Hochschulen noch Höhere Schulen sind, „sie stehen irgendwo dazwischen und unterstehen direkt dem Unterrichtsministerium" (ebd., 101). Mit der Verabschiedung des Pädagogischen Hochschulgesetzes 2007 wurden aus den „Pädagogischen Akademien“ „Pädagogische Hochschulen“. Damit wurde in Österreich die Lehrerausbildung für Volksschulen, Hauptschulen und Sonderschulen und für Berufsschulen auf Hochschulniveau gehoben.

\section{Frankreich}

Auch in Frankreich beginnt die Professionalisierung der Lehrerbildung im frühen 19. Jahrhundert. Es wurden die „écoles normales“ (EN) als staatliche Lehrerbildungsanstalten gegründet (Grundig de Vazquez, 2012, 128). Sie boten eine dreijährige fachliche und pädagogische Ausbildung an, für die die Kandidaten eine Aufnahmeprüfung ablegen mussten. Wie auch in Deutschland war der Volksschullehrer sozial wenig begünstigt, doch die Lehrerbildungsanstalten sorgten zumindest für verbindliche staatliche Leistungsstandards und dafür, dass der Lehrerberuf allmählich durch den regulären Ausbildungsweg aufgewertet wurde. Jede Kommune sollte nun mit einer Volksschule ausgestattet werden. Nach dem Ende des deutsch-französischen Krieges 1870/71 zeichneten sich unter der Regierung der reformorientierten Republikaner bedeutende Fortschritte in der französischen Lehrerbildung und eine grundlegende Erneuerung des französischen Volksschulwesens ab. Die Republikaner „hofften auf eine neue Generation von rational denkenden, republiktreuen, sozial orientierten und gebildeten Bürgern, welche die demokratische Gesellschaft tragen sollten. Dafür war eine flächendeckende hochwertige Primarschulbildung nötig, in deren Genuss jeder einzelne kommen sollte." (ebd., 129). Nicht die soziale Herkunft, sondern die Leistung sollte entscheidend sein. Die Schulen sollten staatlich und kostenfrei sein. Nur ein gut qualifiziertes Lehrpersonal kann diesen Anforderungen gerecht werden. In Frankreich begann man also schon 50 Jahre früher als in Deutschland die Schulen und die Lehrerbildung zu reformieren.

Das bisherige Lehrerbildungssystem wurde um elitäre Lehrerbildungsanstalten ergänzt, wie z.B. die "écoles normales supérieures" (ENS), in denen besonders begabte Lehramtsstudenten auf höhere Posten im Primarstufenbereich vorbereitet werden sollten. 1880 wurden die ersten Schulen für weibliche Lehrkräfte gegründet.

Ähnlich wie in Deutschland sah die Volksschullehrerbildung jedoch vor, dass den Lehrern ein zwar fundiertes, aber eingeengtes Wissen vermittelt werden sollte, das sie intellektuell nicht zu weit von den Schülern entfernte, um deren Bedürfnisse weiterhin wahrnehmen zu können. Das führte dazu, dass die Volksschullehrer in der Regel aus bescheidenen Verhältnissen stammten, was von Regierungsseite erwünscht war. Ein wichtiges Ziel war eine gefestigte moralische Grundhaltung. Die Lehrer des höheren Bildungswesens gingen aus den Universitäten hervor.

1989 wurden einheitliche Lehrerbildungseinrichtungen beschlossen. Diese universitären Lehrerbildungsinstitute „instituts universitaires des formation des maitres“ (IUFM) sind für Ausund Fortbildung aller Lehrkräfte des staatlichen Schulwesens verantwortlich. 1990 wurden die 
Lehrer aller Schulformen auf eine Gehaltsstufe gestellt - ein Vorgang, auf den man in Deutschland wohl noch lange warten muss. 2005 wurden die IUFM an die Universitäten angegliedert (ebd., 133).

\section{England}

Die Lehrerausbildung im 19. Jahrhundert lässt sich als Meisterlehre („class-room based apprenticeship“) bezeichnen. In der zweiten Hälfte des 19. Jahrhunderts gab es das sogenannte „PupilTeacher-System“: Die Schüler erhielten mit 13 Jahren die Möglichkeit in eine 5 Jahre dauernde „Meisterlehre“ aufgenommen zu werden, wobei die fachliche Unterweisung nur einen sehr geringen zeitlichen Anteil hatte. Als Ergänzung konnten sie ein „Training-College“ besuchen, das auf moralische Erziehung und die Erziehung der Persönlichkeit fokussiert war. Zu Beginn des 20. Jahrhunderts gab es für die Lehrer der Sekundarstufe mehr Fachunterricht, damit wurde jedoch der Bezug zur Praxis verringert. Folglich wurde die Vermittlung pädagogischer Techniken an die "Colleges Tutors" transferiert und gehörte nicht mehr zur Profession an sich. Im Laufe der ersten Jahrzehnte des 20. Jahrhunderts war die Ausbildung nicht mehr nur auf einen „Meister“ orientiert und die Lehramtsanwärter konnten selbstbestimmter ihre Ausbildung gestalten.

In den 1950-1970er Jahren gab es eine Verlagerung der Lehrerbildung in die höhere Bildung: "Colleges of education“, „Instituts of higher education“ und "Polytechnics“ stellten solche Programme bereit. Die Einführung des „Bachelors of Education“ brachte eine Reihe neuer Disziplinen wie Soziologie, Psychologie, Philosophie und Geschichte mit sich. Als Ergebnis wurde die Lehrerausbildung zunehmend akademisiert auf Kosten der Praxisanteile und somit verarmte die Beziehung zwischen akademischem Wissen und praktischen Fertigkeiten mehr und mehr. In den 1980er Jahren geriet die Schule wieder in den Blick der Politik und es wurde ein „professional standards framwork" mit einem zentral vorgeschriebenen Curriculum, einem rigorosen Inspektionssystem und der Orientierung auf den Schüler out-put, auf die die Lehrerbildung verpflichtet wurde, eingeführt. Dies führte 1998 zu einem Rahmen für die Standardisierung der Lehrerausbildung (DFFE, QTS). Damit beginnt eine neue Professionalisierung der Lehrerbildung, die Vorbild für alle anderen europäischen Länder wurde.

Heute wird dieses System teilweise als zu technokratisch („highliy technicist, instrumental approach to teaching") kritisiert. Die Kritik führt soweit, dass ein Politiker 2010 die alte Meisterlehre wieder einführen möchte: „Teaching is a craft and it is best learnt as an apprentice observing the master craftsman or woman" (Jones, 2012, 206).

\section{Italien}

In Italien hatten im 19. Jahrhundert die Lombardei und das Königreich Sardinien die besten Bildungsgesetze. Die Lombardei hatte schon im ausgehenden 18. Jahrhundert die gleiche Lehrerausbildung wie Österreich. Daher war die Lombardei für das 1861 vereinte Italien maßgebend für alle anderen Staaten und von hier ausgehend verbreitete sich die „Normalmethode“ (Polenghi, 2012, 139). Die Mehrheit der Lehrer - und im Gegensatz zu Deutschland nicht nur der Gymnasiallehrer, sondern auch der Volksschullehrer - waren Priester, außer in der Lombardei und in Südtirol, als Folge der Politik Josephs II. Auch kamen von dort die weiblichen Lehrkräfte, die es im übrigen Italien zu dieser Zeit noch nicht gab.

Italien wurde 1859 vereint, das bedeutete, dass ein Schulgesetz verabschiedet wurde, das im Königreich Italien bis 1923 in Kraft war. Erst 1913 gingen die Grundschulen von den Kommunen in die staatliche Verantwortung über. Die Normalschule, in der weiterhin die Grundschullehrer für die sechsjährige Grundschule ausgebildet wurden, dauerte nur zwei oder drei Jahre und konnte bereits 
von 14jährigen Schülern besucht werden. 1896 wurde eine dreijährige Mittelschule eingeführt, doch die Ausbildung der Grundschullehrer blieb bis Anfang des 20. Jahrhunderts oberflächlich. „Da Italien viele Lehrer benötigte, um den Analphabetismus (besonders im Süden) zu bekämpfen, zogen die Regierungen Quantität statt Qualität vor“ (ebd., 141). Wie auch in den anderen europäischen Ländern forderten die Lehrer selbst (siehe Lehrergewerkschaft „Unione Magistrale Nazionale“, 1900 gegründet) eine besser Ausbildung. Doch nur einige Universitäten boten zwischen 1904 und 1923 sogenannte zweijährige „Pädagogische Schulen“ an, für diejenigen, die Direktor einer Schule werden wollten. Auf diese Lehramtsstudenten wurde von den anderen Studierenden herabgeschaut, denn „die Universität sollte in ihren Augen elitär bleiben“ (ebd., 141). Da es für Mädchen die einzige Möglichkeit war, eine bessere Bildung zu erwerben, wurde die Normalschule schon um 1900 zu 94 Prozent von Mädchen besucht. Nun waren nicht mehr Priester, sondern Frauen in der Grundschule. Diese rasche Feminisierung hatte zwei pragmatische Gründe: Die Frauen erhielten 1/3 weniger Gehalt als die Männer und sie ließen sich weniger in Gewerkschaften organisieren (ebd., 142). Letztlich war dies jedoch ein wichtiger Weg zur Emanzipation - Maria Montessori (1870-1952) war eine der bedeutendsten Pädagoginnen, die auf diesem emanzipatorischen Weg voranschritt.

1923 wurde zur Regierungszeit Mussolinis die „Pädagogische Schule“ geschlossen, die Normalschulen wurden jedoch reformiert und verbessert, sie hießen jetzt "Istituto Magistrale“. Das Curriculum wurde auf Kosten der Praktika erweitert - wie auch in vielen anderen europäischen Ländern. Erst 1952 wurde in die Grundschullehrerausbildung das Praktikum wieder eingeführt. Nach dem „Istituto Magistrale“ konnte eine vierjährige Pädagogische Fachhochschule besucht werden, die zum Lehramt in der Sekundarstufe befähigte. Gymnasiallehrer mussten ein Studium absolviert haben, und auch hier findet sich wieder die Ansicht, dass Sekundarschullehrer keine pädagogische und didaktische Ausbildung nötig haben (ebd., 145).

Seit 1995 wurde die Pädagogische Fakultät in die Bildungswissenschaftliche Fakultät umgewandelt. Ab 2011 muss für das Grundschullehramt ein 5-jähriger Kurs absolviert werden mit vorheriger Zulassungsprüfung. Dies bedeutet nach Polenghi jedoch keine Verbesserung, „da das Curriculum vom Enzyklopädismus geprägt ist" (ebd., 148). Grundschullehrer müssen jedoch Sonderpädagogik und Klinische Psychologie studieren, da die Integration von Schülern mit einer Behinderung in die Regelschule in Italien obligatorisch ist. Als Besonderheit fügt Polenghi (ebd., 150ff.) noch an, dass in den Grundschulen fast 100 Prozent Frauen unterrichten und der Anteil in der Sekundarstufe steigend ist (damit ist er sehr viel höher als in anderen europäischen Ländern). Das wirkt sich - auch im Vergleich mit anderen Ländern - heute noch negativ auf das Gehalt und den sozialen Status aus.

\section{Analyse und Gesamtüberblick}

Man kann von einer gesamteuropäischen Entwicklung der Institutionalisierung der Lehrerbildung für das niedere Lehramt sprechen, die im ausgehenden 18. Jahrhundert allmählich beginnt. Bis Ende des 19. Jahrhunderts gab es in der Regel flächendeckende Lehrerbildungsstätten für das niedere Lehramt in allen westeuropäischen Ländern, auch den hier nicht näher beschriebenen skandinavischen Ländern. Die gemeinsame Grundlage für eine Professionalisierung der Lehrerbildung ist die Aufklärung, die den selbstständig denkenden Menschen erforderte.

Auch in der Zeit vom ausgehenden 19. Jahrhundert bis zum Ende des ersten Weltkrieges zeigen sich vergleichbare Entwicklungsstränge, ausgelöst durch den Perspektivenwechsel hin zum Kind, seinen Bedürfnissen und individuellen Entwicklungsmöglichkeiten. Reformpädagogische Ansätze, die das Kind in den Mittelpunkt ihrer pädagogischen und didaktischen Bemühungen stellen, finden 
sich in fast allen europäischen Ländern. Allen gemeinsam ist jedoch auch, dass die reformpädagogischen Theorien zwar Eingang in die Lehrerbildung gefunden haben, jedoch eher nicht die Lehrerbildung insgesamt prägten, die nach wie vor von den jeweiligen nationalen politischen Gegebenheiten abhängig war. Die Revolution 1848 wirkte sich z.B. in Deutschland sehr viel anders aus als in Frankreich und die Zeit nach dem ersten Weltkrieg brachte nur in den Ländern, die von der Monarchie in eine Republik übergingen, auch einschneidende Reformen in der Bildungspolitik wie in Deutschland und Österreich. Die Zeit zwischen 1930 und 1945 war insbesondere für Deutschland sowie teilweise auch Österreich und Italien - nicht aber für Frankreich und England - bildungspolitisch relevant.

Gemeinsam ist allen westeuropäischen Ländern auch die Kluft zwischen den beiden Lehrerkategorien: dem Lehramt für die „höhere“ Bildung und dem Lehramt für das „niedere“ Volk. Die einen absolvieren als Lehrerbildner für zukünftige Studenten ein wissenschaftliches Studium (in der Regel ohne oder nur mit geringen pädagogischen Anteilen), die anderen eine praxisorientierte, im Wissenskanon eingeengte Ausbildung im Sinne von: „The man who teaches the children of peasenats must be a peasant himself" (zitiert nach Winter, 1980, 297). Wie viel Fachwissenschaft, wie viel Pädagogik, wie viel Praxis benötigt eine Lehrerausbildung? Diese Diskussion wird in allen Ländern immer wieder neu geführt - auch das ist eine Gemeinsamkeit.

Gegen Ende des zwanzigsten Jahrhunderts entscheiden sich einige Länder wie England und Frankreich für eine einheitliche Lehrerbildung. Roth schreibt dazu: „Volksschullehrerbildung spiegelt deshalb immer, was eine Gesellschaft von dem sogenannten ,Volke' denkt und hält, wieweit Unterricht und Erziehung Herrschaftsausübung ist, wieweit die Beschränkung und Begrenzung der Information für das Volk geht (...), - oder wieweit sie Aufmunterung zur Teilhabe und Partizipation praktizieren will“ (Roth, 1975, 824).

Die Zeit unmittelbar nach dem zweiten Weltkrieg ist generell gekennzeichnet durch den Wiederaufbau - Volksbildung und „höhere“ Bildung waren weiterhin unterschiedliche Ausbildungs-stränge. „Vielfältige Maßnahmen nationaler Bildungspolitik stellen sich auf westeuropäischer Ebene als Veränderungen dar mit den Zielsetzungen, eine allgemeine Höherqualifikation zu realisieren, die ,Chancengleichheit' des Zugangs zu den Bildungsinstitutionen zu schaffen, die Barrieren zwischen unterschiedlichen Schullaufbahnen abzubauen" schreibt Winter $(1980,261)$ und er fährt fort: „Dieser Prozeß, in den 60er Jahren eingeleitet, dauert an und trifft in der Gegenwart auf die verschärft restriktiven Bedingungen einer langfristigen Wirtschaftsund Gesellschaftskrise“" (ebd., 262).

Die nationalen Gesellschaftssysteme können seit Kriegsende ökonomisch und politisch als Subsysteme der Weltwirtschaft und Weltpolitik verstanden werden. Daher sind insbesondere die letzten Jahrzehnte durch einen bisher nicht dagewesenen Internationalisierungsprozess gekennzeichnet. Stärker als bisher bedingen politisch-ökonomische Veränderungen „wechselnde Machtkonstellationen innerhalb des internationalen Systems und damit in dialektischer Abhängigkeit nationale Entwicklungen" (Winter, 1980, 73). Die Schulentwicklung und die Lehrerbildung kann daher nicht mehr nur national betrachtet werden, denn die Tendenz geht zu einer Vereinheitlichung und Vergleichbarkeit innerhalb Europas, um der Globalisierung Rechnung zu tragen. Der Bologna-Prozess, der bis 2010 die Schaffung eines einheitlichen europäischen Hochschulraums vorsah, ist diesem Trend verpflichtet. Grunder bezeichnet ihn als „den folgenschwersten Wandel in der tertiären Ausbildung seit zweihundert Jahren. Die Umstellung von Diplom und Lizentiat auf B.A. und M.A. kommt einer revolutionären Umwälzung gleich. Revolutionen haben Vorteile und Nachteile." $(2010,338)$. Eine differenzierte Diskussion dieser Vorund Nachteile ist an dieser Stelle nicht möglich. In allen Ländern, die sich dem Bologna-Prozess angeschlossen haben, zeigt sich jedoch, dass damit die Reformen keineswegs abgeschlossen sind, 
sondern dass eine Reform die andere ablöst. Ob sich dadurch die Lehrerausbildung verbessert, sei dahingestellt.

Vorteil ist jedoch die Vereinfachung der Internationalisierung in Form von gegenseitiger Anerkennung der Leistungen in Doppelabschlussprogrammen, die Durchführung von internationalen Projekten im Bereich der Curriculumentwicklung (siehe TEMPUS-Projekte der EU) und des teilweise einfacheren Studentenaustauschs.

Der Europäische Qualifikationsrahmen (engl. EQF), eine Initiative der Europäischen Union, trägt dazu bei, Bildungsabschlüsse vergleichbarer zu machen. Er definiert eine Reihe von Bildungsniveaus, bezogen auf Kenntnisse, Fertigkeiten und Kompetenzen. Diese unverbindliche Empfehlung wurde 2008 vom Europäischen Parlament beschlossen. Sie ist ein weiterer Schritt in die Richtung einer europäischen Vereinheitlichung der Lehrerbildung und einer größeren Vergleichbarkeit der Ausbildungssysteme.

\title{
Schlussbemerkung
}

\begin{abstract}
„Aufklärung im Sinne Kants, als Ausgang des Menschen aus selbstverschuldeter Unmündigkeit, Liberalismus und Rechtsstaat, die bürgerliche Bewegung zur Freiheit und zur Nation hin, die Revolution von 1848 und die industrielle Revolution, Kapitalismus und Arbeiterbewegung, die Entwicklung der Wissenschaft und Kunst: Das alles war europäisch ebenso wie deutsch. Was ,Bildung' einmal meinte, war im Ursprung ohnehin europäisch oder vielmehr und ganz entschieden weltbürgerlich angelegt." (von Krockow, 1990, 106)
\end{abstract}

Das Vorhergehende hat viele Gemeinsamkeiten westeuropäischer Lehrerbildung gezeigt wie auch nationale Unterschiede. Eine Bemerkung sei jedoch am Schluss gestattet, die einen Aspekt aufgreift, der bisher nicht erwähnt wurde: die Lehrerpersönlichkeit selbst. Letztlich kommt es auf die Lehrerpersönlichkeit an, auf ihre Befähigung, das Beste in den Kindern zu wecken und sie zu ihren persönlichen Höchstleistungen zu führen und das ist nur zu einem Teil abhängig von Ausbildungssystem und Land. Sandfuchs schreibt zwar völlig richtig: „Bis heute ist die Entwicklung der Lehrerbildung bestimmt von der gesellschaftlichen Einstellung zur Bedeutung von Schulbildung und Lehrberuf, dem Wechsel von Überfüllungs- und Mangelkrisen im Lehrerberuf, den ökonomischen Bedingungen sowie politischen und weltanschaulichen Positionen und Überzeugungen. Sie ist zudem gekennzeichnet durch eine zuweilen nur vordergründig sachliche Diskussion, hinter der bildungspolitische Vorbehalte, finanzielle Erwägungen und Interessen von Statusgruppen verborgen werden" (Sandfuchs, 2012, 62). Doch auch wenn die je nationale Bildungspolitik die Reformen vorgibt, so ist doch der Lehrer nicht willenloses Instrument. Er kann „für eine allseitige Entwicklung der menschlichen Persönlichkeit und den gesamt gesellschaftlichen Fortschritt tätig sein und verhindern, daß aus der reformierten Schule eine formierte wird“ (Winter, 1980, 263).

\section{Literatur}

Blankertz, H. (1982): Die Geschichte der Pädagogik. Von der Aufklärung bis zur Gegenwart. Wetzlar: Büchse der Pandora.

Dresselhaus, G. (1997): Das deutsche Bildungswesen zwischen Tradition und Fortschritt: Analyse eines Sonderwegs. Münster: LIT-Verlag.

Feige, B. (1997): Philanthropische Reformpraxis in Niedersachsen. Köln: Böhlau.

Goebel, K: (1995): Wer die Schule hat, hat die Zukunft. Bochum: Universitätsverlag Dr. N. Brockmeyer. 
Grimm, G., B. Bali \& V. Pirka (2012): Lehrerbildung in Österreich - Aspekte ihrer Genese von den Anfängen im späten 18. Jahrhundert bis zur Zweiten Republik. In: Németh, A. \& Skiera, E. (Hrsg.): Lehrerbildung in Europa. Geschichte, Struktur und Reform. Frankfurt a. M.: Peter Lang, S. 91-110.

Grundig de Vazquez, K. (2012): Die Lehrerbildung in Frankreich - Geschichte, Struktur und Reform. In: Németh, A. \& Skiera, E. (Hrsg.): Lehrerbildung in Europa. Geschichte, Struktur und Reform. Frankfurt a. M.: Peter Lang, S. 123-138.

Grunder, H.-U. (2010): Bologna in der Schweiz: ,neue' Ausbildungskonzepte, ,neues Lernen'? In: Graumann, O. et al. (Hrsg.): Hochschule und Schule in der internationalen Diskussion. Baltmannsweiler: Schneider, S. 338-346.

Jones, M. (2012): Teacher education in England. In: Németh, A. \& Skiera, E. (Hrsg.): Lehrerbildung in Europa. Geschichte, Struktur und Reform. Frankfurt a. M.: Peter Lang, S. 201-218.

Kant, I. (1784): Was ist Aufklärung? In: Kant, I.: Werke, hrsg. von E. Cassirer. Berlin: Reimer 19211923, Bd. IV.

Keck, R. W. (1989): Die Entwicklung der Lehrerbildung in Deutschland im 18. und 19. Jahrhundert. In: Prinz v. Hohenzollern \& Liedke, M. (Hrsg.): Schreiber, Magister, Lehrer. Zur Geschichte und Funktion eines Berufsstandes. Bad Heilbrunn: Klinkhardt, S. 195-213.

Keck, R. W. (1997): Schulbildung im Lichte des europäischen Scholastizismus: eine Geschichte der Instrumentalisierung oder Vervollkommnung? In: Lüth, C. \& Wulf, C. (Hrsg.): Vervollkommnung durch Arbeit und Bildung? Weinheim: Beltz, S. 183-192.

Keck, R.W. (2002): Schulwesen. In: Schneider, H. \& Landfester, M. (Hrsg.): Der Neue Pauly. Enzyklopädie der Antike. Band 15/2. Stuttgart, Weimar: J.B. Metzler, S. 1110-1115.

Keck, R.W. (2003): Konfessionalisierung und Bildung aus erziehungswissenschaftlicher Sicht. In: Musolff, H.-U. \& Göing, A.-S. (Hrsg.): Anfänge und Grundlegungen moderner Pädagogik im 16. und 17. Jahrhundert. Köln: Böhlau Verlag, S. 11-30.

Keck, R. W. (2007): Schulgeschichtliche Entwicklung in der Frühen Neuzeit. In: Jacobi, J. (Hrsg.): Zwischen christlicher Tradition und Aufbruch in die Moderne. Tübingen: Max Niemeyer, S. 13-28.

Keck, R. W. (2009): Zur Geschichte der Schule. In: Blömeke, S. (Hrsg.): Handbuch Schule. TheorieOrganisation-Entwicklung. Bad Heilbrunn: Klinkhardt, S. 157-162.

Krockow, C. Graf von (1990): Die Deutschen in ihrem Jahrhundert 1890-1990. Hamburg: Rowohlt.

Marx, K. \& Engels, F. (1976): Werke. Band I. Berlin: Karl Dietz Verlag.

Németh, A. (2012): Lehrerbildung in Ungarn. In: Németh, A. \& Skiera, E. (Hrsg.): Lehrerbildung in Europa. Geschichte, Struktur und Reform. Frankfurt a. M.: Peter Lang, S. 21-39.

Oelker, J. \& Neumann, D. (1985): Ausbildungskonzepte für den Primarstufenlehrer. In: Lenzen, D. (Hrsg.): Enzyklopädie Erziehungswissenschaft. Band 7: Erziehung im Primarschulalter (hrsg. von K. Hemmer \& H. Wudtke) Stuttgart: Klett-Cotta, S. 126-137.

Polenghi, S. (2012): Die Lehreraubildung in Italien. In: Németh, A. \& Skiera, E. (Hrsg.): Lehrerbildung in Europa. Geschichte, Struktur und Reform. Frankfurt a. M.: Peter Lang, S. 139-155.

Roth, H. (1975): 100 Jahre Lehrerbildung. In: Die Deutsche Schule, 67 (12), S. 819-831.

Sandfuchs, U. (2004): Geschichte der Lehrerbildung in Deutschland. In: Blömeke, S. et al. (Hrsg.): Handbuch Lehrerbildung. Bad Heilbrunn: Klinkhardt, S. 13-37.

Sandfuchs, U. (2009): Johannes Kühnel. Ein Seminar- und Reformpädagoge. In: Sandfuchs, U., Link, J.-W. \& Klinkhardt, A. (Hrsg.): Verlag Julius Klinkhardt 1834-2009. Bad Heilbrunn: Klinkhardt, S. 58-79.

Weiner, H. (1992): Geschichte der Pädagogik. Berlin: De Gruyter.

Winter, K. (1980): Das Europäische Bildungswesen im Prozeß seiner Internationalisierung. Weinheim und Basel: Beltz Verlag. 
Graumann: Aspekte der Geschichte westeuropäischer Lehrerbildung International Dialogues on Education, 2014, Volume 1, Number 1, pp. 42-58 ISSN 2198-5944

\section{Über die Autorin}

Prof. em. Dr. Dr. Olga Graumann ist Professorin für Schulpädagogik und Beauftragte für internationale Kooperation an der Universität Hildesheim. 